\title{
A Magnitude Limited Redshift Survey near the SGP
}

\author{
Quentin A. Parker ${ }^{1}$ \\ Anglo-Australian Observatory, Coonabarabran, NSW 2357, Australia
}

\begin{abstract}
A description of a magnitude limited redshift survey covering 9 adjacent UKST fields is presented together with some initial results. The survey is currently $\sim 90 \%$ complete to $b_{j} \leq 17.1$. The compilation of $\sim 1700$ redshifts will form one of the largest, independent, contiguous, well sampled and most homogeneous redshift catalogues currently available. The special features of this catalogue are its coverage $\left(\sim 330 \mathrm{deg}^{2}\right)$, mean sampling depth $\left(\sim 125 h^{-1} \mathrm{Mpc}\right)$ and field to field photometric accuracy $\left(b_{j} \pm 0.05\right)$. Morphological classifications have also been determined for all galaxies in the survey fields down to $b_{j} \leq 17.1$ and COSMOS image parameters are also available. This extensive catalogue should prove a valuable database for many projects but particularly in delineating the detailed structure in the large scale galaxy distribution.
\end{abstract}

\section{The Survey}

A large redshift survey near the SGP to $b_{j} \leq 17.1$ has been compiled over the last 7 years using the SAAO $1.9 \mathrm{~m}$ telescope and latterly the multi-object fibre spectroscopy system, 'FLAIR', on the UKST (Parker \& Watson 1990, 1994; Watson \& Parker 1994 ). So far 1700 galaxy observations have been obtained which should yield a completeness level of $\sim 90 \%$ to $b_{j} \leq 17.1$. Of these observations $\sim 1000$ have been made with FLAIR and the rest with the $1.9 \mathrm{~m}$ at SAAO. FLAIR offers a more efficient means of performing surveys of this nature (Watson 1988) and has led to a doubling of the survey over 3 observing runs during 1992/93. A contiguous 3 hour strip in RA at a constant declination of $-35^{\circ}$ has been sampled covering the 9 UKST SERC survey fields $406,407,408$, $349,350,351,352,353$ and 354 . The 'continuous strip' observing strategy is not only an observationally efficient slice to adopt but should also be more sensitive to large-scale feature detection than a simple square area of the same solid angle. The mean depth of the current survey is $\sim 125 h^{-1} \mathrm{Mpc}$ but sampling to a depth $\geq 250 h^{-1} \mathrm{Mpc}$ is achieved. The object database has been obtained from COSMOS machine measures of UKST/SERC $\mathrm{J}$ survey plates of the 9 fields of interest. Star-galaxy separation of the COSMOS data is performed using a geometric discriminator which has proved to be highly successful $(\sim 95 \%)$ at these magnitudes (MacGillivray \& Stobie 1984). Visual examination of the images of all COSMOS 'classified' galaxies to $b_{j}=17.1$ in each field has revealed

\footnotetext{
${ }^{1}$ Currently on special leave from the Royal Observatory Edinburgh, UK
} 
a modest $\sim 7 \%$ contamination by multiple stars and spurious images for the entire survey area. Furthermore the well calibrated Edinburgh Durham Southern Galaxy Catalogue (EDSGC) magnitudes are used (Heydon-Dumbleton et al. 1989) which ensure the consistency of field to field magnitude limits to \pm 0.05 giving a homogeneous sample. Effects on the contribution to $\xi(r)$ from different fields due to magnitude limit inconsistencies should thus be small. Morphological classifications for all galaxies in the survey have also been obtained from visual scrutiny of the UKST plate material. Independent classifications of the galaxy samples in each field were made by the author and three different summer students before an average classification was adopted. The classification scheme followed the de Vaucouleurs extension to the Hubble sequence. The homogeneity and completeness of the survey should permit a detailed statistical description, determination of group and cluster membership and allow the tracing of inter-connected features over adjacent fields.

The adopted magnitude limit of this survey is compatible not only with the Durham AAT SAAO redshift survey (Shanks et al. 1989) but also with the new 'redshift map of 4000 galaxies' currently being constructed with the FLAIR system on the UKST which covers the 60 fields of the EDSGC at a 1 in 3 sampling rate to $b_{j} \leq 17.0$ (see Broadbent et al. in these proceedings). A rigorous and independent comparison can be made between the three surveys.

\section{Survey aims}

The overall aims of this survey are quite broad but include:

To obtain a 3-D description of the galaxy distribution sufficient in depth and across the line of sight to intersect many "scale-lengths" of typical void dimensions so that their properties can be characterised and so that a more representative 'fair' sample of the Universe is obtained.

To determine the 2-pt spatial correlation function for our sample of $\sim 700$ galaxies in contiguous fields out to $\sim 200 \mathrm{~h}^{-1} \mathrm{Mpc}$ to determine both the slope of the power law and the correlation length as these constrain the possible spectrum of primordial density fluctuations on large scales (Peebles 1980). At these depths the influence of the LSC on the form of the correlation function should also be small. Confirmation of the 'shoulder' feature seen in $\xi(r)$ between $1-7 h^{-1} \mathrm{Mpc}$ (Shanks et al. 1989) should be possible. The verification of such a feature could imply non-scale-free clustering at small scales.

To form an intermediate comparison sample between the shallower but wide angle coverage of the extended CfA survey and the narrow but very deep pencil beam surveys in the hope of learning something about galactic evolution.

To investigate the relative space densities of galaxies of different morphological types, variations in their luminosity functions and possible segregation in galaxy groups and the occurrence of emission lines in their spectra as these too have a bearing on galactic evolution and environmental influences.

To obtain a quantitative description of the observed galaxy sample into groups, associations and field galaxies using techniques of hierarchical clustering for studying the properties of small scale structure. 


$$
\begin{gathered}
22.5<\mathrm{RA}<2.3 \\
-38<\mathrm{DEC}<-32
\end{gathered}
$$

RA

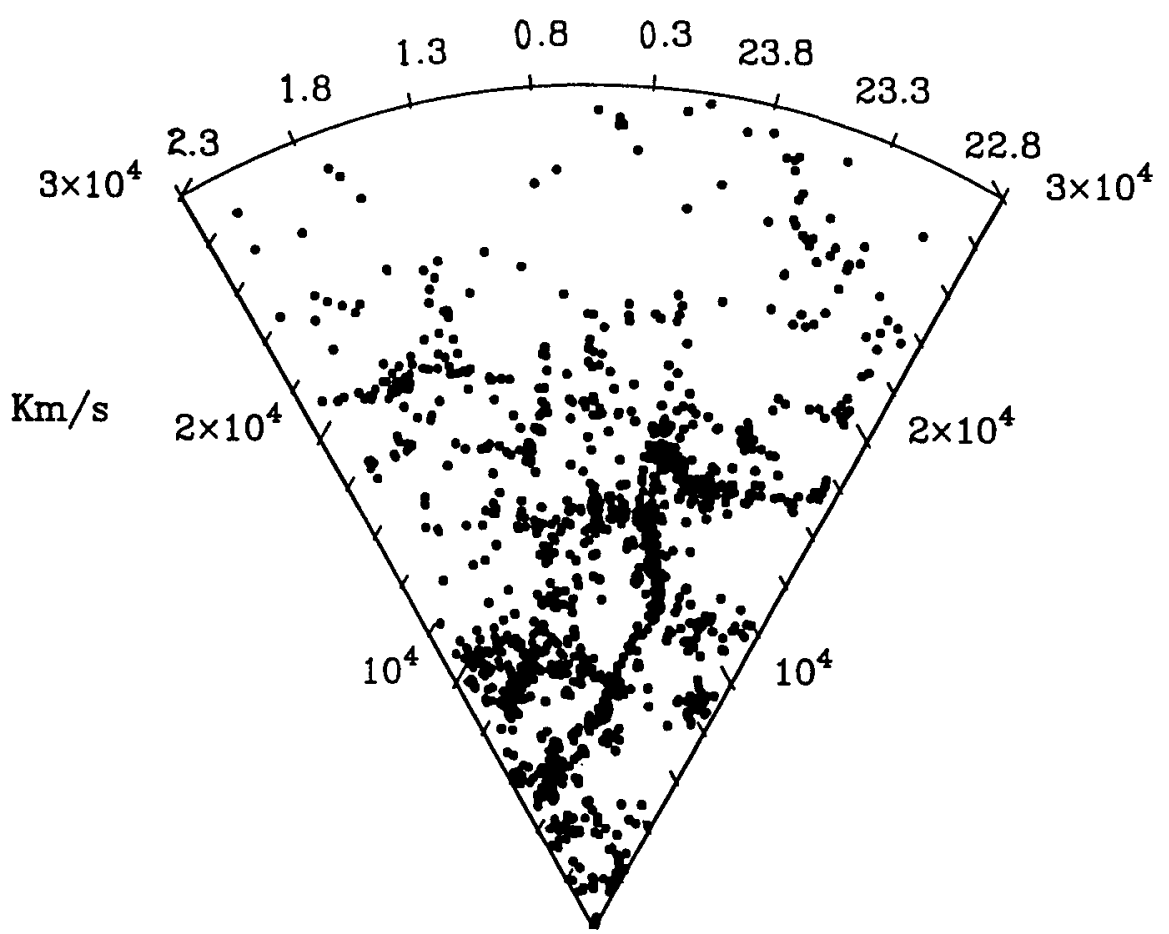

Figure 1. Preliminary cone diagram of $\sim 1400$ galaxy redshifts over 9 contiguous fields of the SGP survey area to $B_{j} \leq 17.1$. 

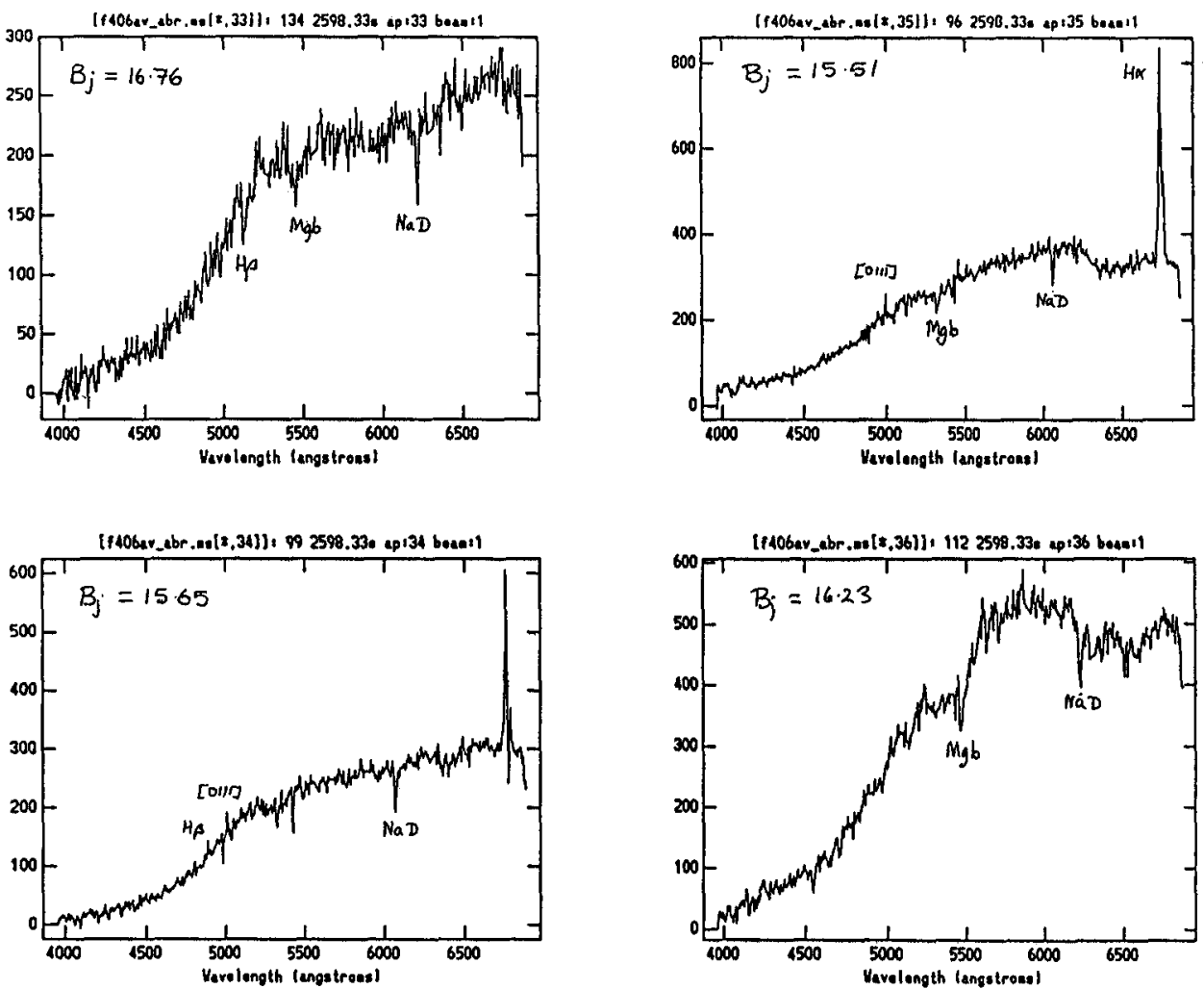

Figure 2. Consecutive reduced fibre spectra of four galaxies in field 406 of the survey. 
The results for this deep survey can also be compared with the results from planned deeper surveys such as the ESO key program of de Lapparent et al. who aim to obtain $\sim 700$ galaxy redshifts in a $0.4 d^{2} g^{2}$ area to $R \leq 20.5$.

\section{Preliminary results}

Redshifts for 107 galaxies in field 349 are given by Parker et al. (1986), whilst 116 redshifts in field 352 are given by Parker and Watson (1990). A catalogue of the complete redshift survey of $\sim 1700$ galaxies over the 9 contiguous fields is currently in preparation. A cone diagram of right ascension and redshift from the fully reduced data available so far to $b_{j} \leq 17.1$ is given in Fig. 1. It contains $\sim 1400$ redshifts over the 9 fields in the survey. The wedge is $\sim 6^{\circ}$ thick in declination space. Significant voids are revealed along with various well defined galaxy groups and "sheets" which have only a limited velocity dispersion (typically $\leq 200 \mathrm{kms}^{-1}$ ). The cone diagram reveals the interconnected nature and extent of these features. Structure is traced out well to $\sim 200 h^{-1} \mathrm{Mpc}$, $\sim 2 \times$ the depth of the CfA survey. Typical void dimensions are $\sim 20-50 h^{-1}$ $\mathrm{Mpc}$, in good agreement with the current wider angle, though shallower, redshift surveys. Fig. 2. gives examples of four reduced galaxy spectra recently taken with FLAIR in field 406 of the survey. The spectra are from consecutive fibres on the slit and are the result of a total of 4.7 hours integration.

The complete survey of $\sim 1700$ redshifts will provide a valuable database for characterising these structures on larger $\left(\sim 200 h^{-1} \mathrm{Mpc}\right)$ scales. The detailed study of the galaxy distribution in the survey area awaits the satisfactory completion of the full catalogue.

The morphological mix of galaxies between fields in the survey is fairly consistent ( $\sim 55 \% \mathrm{~S} / \mathrm{Irr}$ and $45 \% \mathrm{E} / \mathrm{SO}$ with a $5 \%$ variance) except for field 408 which exhibits a marked $(\sim 60 \%)$ preponderance for ellipticals. The overall percentages compare well with the results from the Durham/AAT redshift survey (DARS), which found $57 \% \mathrm{~S} / \mathrm{Irr}$ and $43 \% \mathrm{E} / \mathrm{SO}$ 's types. Their survey of 330 field galaxies, also based on COSMOS data, has a similar magnitude limit $\left(b_{j} \leq 16.75\right)$ though it consists of 5 discrete fields. The percentage of galaxies exhibiting detectable emission lines in this survey of $\sim 47 \%$ also compares well with $\sim 53 \%$ for the DARS survey.

\section{References}

Heydon-Dumbleton N. H., Collins C. A. \& MacGillivray H. T., 1989, MNRAS, 238,379

MacGillivray H. T. \& Stobie R. S., 1984, Vistas in Astr., 27, 433

Parker Q. A., MacGillivray H. T., Hill P. W. \& Dodd R. J., 1986, MNRAS, 220, 901

Parker Q. A. \& Watson F. G., 1990 A\&AS, 84, 445

Parker Q. A. \& Watson F. G., 1994, in Astronomy from Wide-Field Imaging, Proc. IAU Symp. 161, in press

Peebles P. J. E., 1980, The Large-Scale Structure of the Universe, Princeton University Press 
Shanks T., Stevenson P. R. F., Fong R. \& MacGillivray H.T., 1984, MNRAS, 206,767

Shanks T., Hale-Sutton D., Fong R. \& Metcalfe N., 1989, MNRAS, 237, 589

Watson F. G., 1988, in Fiber Optics in Astronomy, ASP Conf. Ser. 3, S. Barden, ed., p. 125

Watson F. G., \& Parker Q. A., 1994, Proc. SPIE Conf., in press 